\title{
An asymmetry in apparent motion of kinetic objects
}

\author{
K. PRAZDNY \\ Artificial Intelligence Center, FMC Corporation, Santa Clara, California
}

\begin{abstract}
A kinetic object defined by static random noise against the background of dynamic noise was readily seen in apparent motion with another such object. The converse, however-the motion of a figure defined by dynamic noise against the background of static noise-was not perceived with steady fixations. The explanation for this asymmetry may lie in the visual system's stratification tendencies, which position spatiotemporally uncorrelated textures behind correlated regions.
\end{abstract}

Under the appropriate spatial and temporal presentation conditions, visual elements presented sequentially can produce the impression of smooth, uninterrupted motion. Papert (1964) demonstrated that such motion can be seen when the objects are cyclopean (i.e., defined solely by binocular disparity), even when the successive randomdot stereograms have uncorrelated textures (of course, the left and right images have to be correlated in each snapshot to define binocular disparity). Papert's was probably the first experiment indicating that motion can be perceived in the absence of a relevant luminance-domain motion signal. Ramachandran, Rao, and Vidyasagar (1973) reported that motion can be detected when only texture discontinuities are available as input. Petersik, Hicks, and Pantle (1978) showed that apparent motion can be perceived between kinetic objects, that is, objects defined by the differences in spatiotemporal correlation. These experiments show conclusively that this kind of apparent motion cannot be mediated by luminance-domain motion detectors with large receptive fields (see, e.g., Burr \& Ross, 1986, for such recent suggestions).

Here we report a curious asymmetry in apparent motion perceptions based on the discontinuities in spatiotemporal correlations. Although a kinetic figure defined by static noise against the background of dynamic noise (or coherent motion) is readily seen in motion with another such figure, a figure defined by dynamic (snow) noise against the background of static noise is perceived as moving only when the distances are small. When the distances are large, the two figures are seen to "flicker" on and off as they appear from and merge into their background.

\section{METHOD}

The displays were random-dot cinematograms, 256 pixels on each side. The cinematograms were generated by a Symbolics 3640 Lisp machine and displayed on a high-resolution (black-and-white) noninterlaced CRT monitor with fast (P4) phosphor. From $75 \mathrm{~cm}$, the display subtended about $6.25^{\circ}$ of arc $\left(1\right.$ pixel $\left.=1.5^{\prime}\right)$. The randomly placed dots were black or white with equal probability. The cinematograms consisted of a repeating sequence of two subsequences. Each subsequence

The author's address is: Artificial Intelligence Centre, FMC Corporation, 1205 Coleman Ave., P.O. Box 580, Santa Clara, CA 95052. (conceptually equivalent to a "snapshot") defined a kinetic object at a different position and orientation. The duration of each subsequence was controlled by the number of frames in the subsequence (this number could be varied between 5 and 10). Each computer-generated movie frame within a subsequence was presented for $66 \mathrm{msec}$. The interframe interval, as well as the intersubsequence interval, was zero. The kinetic objects were $80 \times 20$ pixel rectangles. The two objects were positioned in diagonally opposite corners of the display (Figure 1). Their centerto-center distance was 150 pixels $\left(3.75^{\circ}\right.$ of arc). There were two random noise conditions: static noise inside the object against dynamic noise in the background, and dynamic noise inside the object against the background of static noise. The subjects were 5 psychophysically experienced observers well acquainted with the phenomenon of apparent motion but naive as to the purpose of the experiment. They were instructed to fixate the center of the display, which was marked by a small black dot. Eye movements were not monitored. The subjects' task was to report the presence or absence of motion. Each subject was presented with 10 displays. The direction of motion (i.e., the diagonal) was chosen at random by computer. The presentation time was about $20 \mathrm{sec}$. Prior to the formal experiment the subjects were shown, and all readily perceived, apparent motion of objects defined in the luminance domain.

\section{RESULTS}

The results were uniform and unequivocal. When the kinetic figure was defined by dynamic random noise against static distribution of random dots, no observer

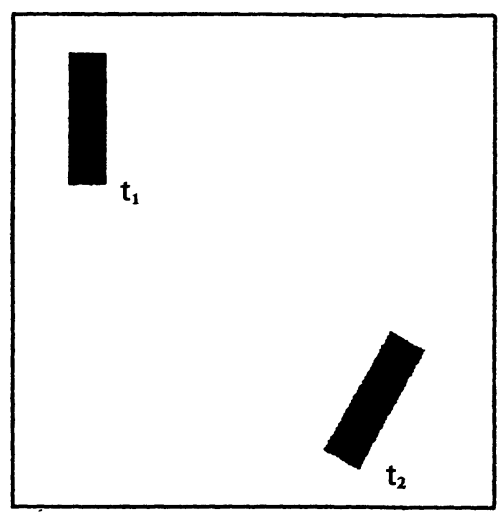

Figure 1. Spatial positions of the two objects in Experiment 1. The two objects were presented sequentially. Object in the upper left corner was presented first, object in the lower right corner second, and so on. In the experiment, black regions were filled with dynamic or static and white regions with static or dynamic noise, respectively. 
reported motion between the two objects when the object separation was large. The objects were reported as successively appearing and disappearing at their positions. When the objects were defined by static noise (different inside the two objects; i.e., the same within each subsequence but different across the two subsequences) against the background of dynamic noise (or coherent, e.g., left-to-right, motion), all observers reported clear perception of apparent motion. These results were, within the parameter range used in this experiment, independent of the number of frames in the subsequence (i.e., the stimulus onset asynchrony).

To investigate this surprising and unexpected result further, a second experiment was performed, using rotating objects with the angle of rotation between the two kinetic objects as the independent variable (Figure 2). The dependent variable was the proportion of time apparent movement, as opposed to sequential appearance and disappearance of objects, was seen. The viewing time was $60 \mathrm{sec}$. All other parameters were the same as in the first experiment. The results are shown in Table 1. As can be seen, when the objects were defined by dynamic random noise against static noise background, the perception of apparent movement was more likely to occur than the perception of sequentiality when the rotation angle was less than $60^{\circ}$. These results should, however, be interpreted with caution. Nearly all observers reported that when they moved their heads with respect to the display, they almost always perceived apparent movement, independently of how the figures were defined. On the other hand, it was possible to see the sequentiality (instead of motion) when the effort was made to carefully maintain the fixation even when the rotation angles were rather small (e.g., $30^{\circ}-$ $\left.45^{\circ}\right)$. It is therefore possible that the perception of motion with small rotation angles was caused by the failure to fixate, that is, that reflex eye movements (captured by the sequential appearance and disappearance of the two objects) were involved. This interpretation is strengthened

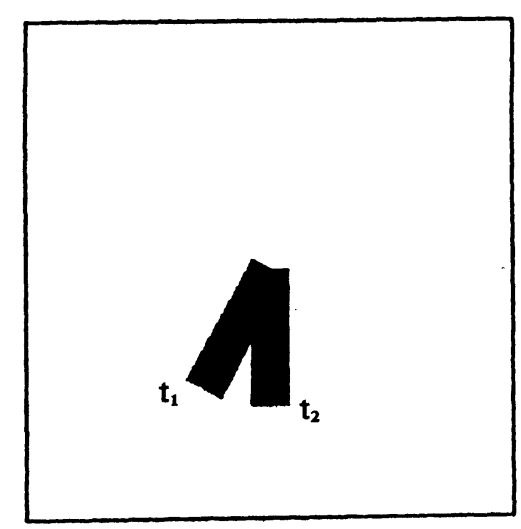

Figure 2. Spatial positions of the two objects in Experiment 2. The two objects were presented sequentially. Vertical object was presented first, rotated object second, and so on. In the experiment, black regions were filled with dynamic or static and white regions with static or dynamic noise, respectively.
Table 1

Percentage of Time of Perceiving Motion (as Opposed to Sequentiality) as a Function of the Rotation Angle in the Experiment Schematized in Figure 2

\begin{tabular}{lrrrrrr}
\hline & \multicolumn{6}{c}{ Rotation Angle } \\
\cline { 2 - 7 } & $15^{\circ}$ & $30^{\circ}$ & $45^{\circ}$ & $60^{\circ}$ & $75^{\circ}$ & $90^{\circ}$ \\
\hline DFSS & 93 & 95 & 89 & 51 & 11 & 7 \\
SFDS & 100 & 100 & 100 & 100 & 100 & 100 \\
\hline
\end{tabular}

Note-DFSS denotes dynamic noise figure within static noise surround. SFDS denotes static noise figure within dynamic noise surround.

by the observation that apparent motion of dynamic noise figures could be seen even over the large distances used in the first experiment when the viewer moved the eyes between the two object locations in synchrony with the object's appearance at one and disappearance at the other location, and that this motion percept disappeared when such tracking stopped.

\section{DISCUSSION}

These results suggest that appropriate spatiotemporal parameters and a clear perception of two successive segmented and well-defined objects are not, in themselves, sufficient for the perception of apparent motion. In the present experiments the two shapes were easily perceived. The perceived asymmetry may have something to do with the subjective impressions observers spontaneously reported when viewing two successive superimposed kinetic objects defined by dynamic noise against the background of static noise. The successive appearance/disappearance of the objects has a depth component. This depth component is absent when the correlation difference is that of static noise against the dynamic noise (or coherently moving) background. It is thus possible that the failure to establish apparent motion between objects defined by dynamic noise against the background of static noise is connected with the visual system's tendency to see spatiotemporally correlated regions "above" the uncorrelated regions. ${ }^{1}$ This tendency may be introducing a signal that interferes with cortical motion detection.

\section{REFERENCES}

BURR, D. C., \& Ross, J. (1986, July). Visual processing of motion. Trends in Neuroscience, pp. 304-307.

PAPERT, S. (1964). Stereoscopic synthesis as a technique for localizing visual mechanisms. MIT Quarterly Progress Report, 73, 239-243.

Petersik, J. T., Hicks, K. I., \& Pantle, A. J. (1978). Apparent movement of successively generated subjective figures. Perception, 7, 371-383.

Prazdny, K. (1985). On the nature of inducing forms generating perceptions of illusory contours. Perception \& Psychophysics, 37, 237-242.

Ramachandran, V. S., Rao, V. M., \& Vidyasagar, T. R. (1973). Apparent movement with subjective contour. Vision Research, 13, 1399-1401.

Wong, E., \& Weisstein, N. (1985). A new visual illusion: Flickering fields are localized in depth plane behind nonflickering fields. Perception, 14, 13-17.

\section{NOTE}

1. Flickering regions (which represent perfect "uncorrelation") are always perceived to lie behind nonflickering regions (Prazdny, 1985; Wong \& Weisstein, 1985).

(Manuscript received for publication December 28, 1986.) 